\title{
ORIGINAL
}

\section{POLÍTICAS REGULADORAS Y OPINIÓN PÚBLICA: EL CASO DEL TABACO}

\author{
Esteve Saltó (1,2), Joan R Villalbí (2,3), Araceli Valverde (2), Lucía Baranda (4) y Antoni Plasencia (2) \\ (1) Consell Asesor de Tabaquisme, Departament de Salut \\ (2) Direcció General de Salut Pública, Departament de Salut \\ (3) Agència de Salut Pública de Barcelona \\ (4) Fundació Jordi Gol i Gurina
}

\section{RESUMEN}

Fundamento: Obtener y difundir información de la opinión pública acerca de un proyecto de regulación permite hacer visible el apoyo social silencioso y facilita el proceso, que con frecuencia debe afrontar resistencias por las partes interesadas. Este trabajo presenta una encuesta de opinión sobre el proyecto de regulación del tabaquismo en los últimos tramos del proceso y sus resultados, y se formulan algunas consideraciones sobre el uso de este tipo de información en procesos de cambio.

Métodos: Estudio descriptivo transversal. En diciembre de 2005 se realizó una encuesta telefónica breve mediante cuestionario precodificado a una muestra de la población de 18 o más años $(n=830)$ de Cataluña. El cuestionario explora la opinión acerca de las propuestas de regulación en discusión. Se presenta el grado de acuerdo y la valoración con nueve medidas regulatorias, estratificando según el patrón de uso de tabaco de los respondientes.

Resultados: El 26,3\% de la población entrevistada son fumadores diarios. El grado de conocimiento y aceptación de la regulación es muy elevado. Los aspectos con mayor apoyo son la prohibición de la venta a menores $(97,3 \%)$, la prohibición de fumar en lugares públicos cerrados $(92,8 \%$ ) y centros de trabajo (89\%), y la prohibición de la publicidad $(90,8 \%)$. El aspecto con menor apoyo es la prohibición de fumar en bares y cafeterías $(80,2 \%)$

Conclusiones: La regulación recibe el apoyo mayoritario de la población. Aunque expresen menos apoyo, las medidas son mayoritariamente aceptadas por los fumadores: son rechazadas mayoritariamente sólo las limitaciones en bares y cafeterías

Palabras clave: Tabaquismo. Contaminación por humo de tabaco. Políticas de salud. Organización y Políticas Gubernamentales.

Correspondencia:

Joan R Villalbí

Agència de Salut Pública de Barcelona

Pl Lesseps 1

08023 Barcelona

Correo electrónico: jrvillal@aspb.es
ABSTRACT

\section{Regulatory policies and public opinion: the case of smoking}

Background: Collecting and disseminating information about the public opinion on a regulatory process gives visibility to the silent social support and facilitates the process, which often confronts resistance from interest groups. This paper presents a survey about a proposed legislation on tobacco in its final stages and its results, and some considerations on the use of this sort of information in change processes.

Methods: Cross sectional descriptive study. In December 2005 a brief telephone survey was made to a population sample of 18 and more years of age $(\mathrm{N}=830)$ in Catalonia (Spain). The questionnaire explored opinions on the proposed regulations under discussion. We present the degree of support and the rating of nine regulatory measures, stratified by the respondents use of tobacco.

Results: Daily smokers are $26.3 \%$ of the surveyed population. Awareness and general support for tobacco regulation are very high. Aspects with wider support include bans on sales to minors (97.3\%), smoking in enclosed public places $(92.8 \%)$ and workplaces $(89 \%)$, and publicity $(90.8 \%)$. The aspect with less social support is banning smoking in bars and cafes $(80.2 \%)$.

Conclusions: The population supports widely tobacco regulation. This reinforces the process and weakens the arguments of those against it. Although smokers are less supportive, most of them accept the regulations, except for limitations in bars and cafes.

Key words: Smoking. Government Policies and Organization. Environmental tobacco Smoke 


\section{INTRODUCCIÓN}

Las políticas regulatorias a favor de la salud suelen afrontar resistencias por parte de los grupos de interés afectados ${ }^{1}$. En una sociedad democrática, la visibilidad del apoyo social favorece la adopción de regulaciones. Esta visibilidad se expresa a menudo en los medios de comunicación, que por tanto juegan un papel importante en tal proceso político $^{2,3}$. Cuando el apoyo social es poco visible, los poderes públicos interesados en la regulación afrontan más dificultades. Es por ello que obtener y difundir información sobre la opinión pública acerca de un proyecto de regulación permite hacer visible el apoyo social silencioso y facilita el proceso.

Recientemente se ha aprobado la Ley de medidas sanitarias frente al tabaquismo en las Cortes españolas ${ }^{4}$. La industria tabaquera $\mathrm{y}$ sus aliados han mantenido un esfuerzo intenso para obstaculizarla y debilitarla, que continua tras su entrada en vigor para difi-

\section{Tabla 1}

Aceptación y valoración media (en una escala de 1 a 10) de diversas medidas de regulación del tabaquismo. Cataluña, 2005

\begin{tabular}{|c|c|c|c|c|c|}
\hline Medida & $\begin{array}{c}\% \text { en } \\
\text { desacuerdo } \\
(1+2+3+4)\end{array}$ & $\begin{array}{c}\% \text { de } \\
\text { acuerdo } \\
(5+6)\end{array}$ & $\begin{array}{c}\% \text { muy de } \\
\text { acuerdo } \\
(7+8+9+10)\end{array}$ & Total & $\begin{array}{l}\text { Valoración } \\
\text { media* }\end{array}$ \\
\hline $\begin{array}{l}\text { Prohibición de la publicidad del tabaco } \\
\text { en todos los medios de comunicación }\end{array}$ & 9,2 & 11,4 & 79,4 & 100,0 & 8,37 \\
\hline $\begin{array}{l}\text { Prohibición del patrocinio de eventos } \\
\text { deportivos, musicales o culturales por } \\
\text { parte de las empresas tabaqueras }\end{array}$ & 16,0 & 15,4 & 68,5 & 100,0 & 7,53 \\
\hline $\begin{array}{l}\text { Prohibición de la venta de tabaco a } \\
\text { menores de } 18 \text { años }\end{array}$ & 2,7 & 1,7 & 95,7 & 100,0 & 9,55 \\
\hline $\begin{array}{l}\text { Limitación de fumar en el lugar de } \\
\text { trabajo }\end{array}$ & 11,0 & 15,8 & 73,2 & 100,0 & 7,97 \\
\hline Limitación de fumar en los restaurantes & 15,7 & 17,9 & 66,3 & 100,0 & 7,49 \\
\hline $\begin{array}{l}\text { Limitación de fumar en los bares y } \\
\text { cafeterías }\end{array}$ & 19,8 & 21,5 & 58,7 & 100,0 & 6,97 \\
\hline $\begin{array}{l}\text { Limitación de fumar en lugares públicos } \\
\text { cerrados como galerías comerciales, } \\
\text { centros culturales, deportivos o de ocio } \\
\text { etc }\end{array}$ & 7,3 & 11,4 & 81,4 & 100,0 & 8,50 \\
\hline $\begin{array}{l}\text { Establecimiento de sanciones para los } \\
\text { responsables de empresas que no hagan } \\
\text { respectar la ley }\end{array}$ & 16,8 & 17,5 & 65,7 & 100,0 & 7,36 \\
\hline $\begin{array}{l}\text { Establecimiento de sanciones para las } \\
\text { personas fumadores que no cumplan la } \\
\text { ley }\end{array}$ & 18,2 & 12,4 & 69,3 & 100,0 & 7,48 \\
\hline
\end{tabular}


cultar su aplicación efectiva ${ }^{5}$. Para contrarrestar este proceso, la Dirección General de Salud Pública del Departamento de Salud de Cataluña, con el apoyo del Consell Assessor de Tabaquisme, entre otras acciones encargó una encuesta dirigida a obtener la opinión de la sociedad catalana al respecto en los últimos tramos del proceso. En este trabajo se presenta esta encuesta y sus resultados, y se formulan algunas consideraciones sobre el uso de la información en procesos de cambio como éste.

\section{SUJETOS Y MÉTODOS}

Se realizó un estudio descriptivo de diseño transversal. Entre los días 2 y 12 de diciembre de 2005 se realizó una encuesta telefónica breve mediante cuestionario precodificado a una muestra de la población catalana de 18 o más años $(n=830)$ por la Fundación Jordi Gol i Gurina, que encargó el trabajo de campo a Promofon SAU. El cuestionario utilizado, basado en buena parte en el utilizado en encuestas previas sobre tabaquismo por la Dirección General de Salud Pública, permitía obtener datos socio-demográficos y relativos al consumo de tabaco de los entrevistados (los lectores interesados pueden solicitar el cuestionario a la Unidad de Tabaquismo de la Dirección General de Salud Pública). También permitía conocer su opinión acerca de diversos componentes de las propuestas de regulación en discusión (se identifican nueve medidas de regulación

Tabla 2

Valoración media (en una escala de 1 a 10) de diversas medidas de regulación del tabaquismo según el status tabáquico. Cataluña, 2005

\begin{tabular}{|c|c|c|c|c|}
\hline Medida & Fumadores & Exfumadores & No fumadores & Total \\
\hline $\begin{array}{l}\text { Prohibición de la publicidad del tabaco en todos los } \\
\text { medios de comunicación }\end{array}$ & 8,16 & 8,24 & 8,58 & 8,37 \\
\hline $\begin{array}{l}\text { Prohibición del patrocinio de eventos deportivos, } \\
\text { musicales o culturales por parte de las empresas } \\
\text { tabaqueras }\end{array}$ & 7,10 & 7,52 & 7,79 & 7,53 \\
\hline $\begin{array}{l}\text { Prohibición de la venta de tabaco a menores de } 18 \\
\text { años }\end{array}$ & 9,52 & 9,44 & 9,63 & 9,55 \\
\hline Limitación de fumar en el lugar de trabajo & 6,67 & 7,95 & 8,74 & 7,97 \\
\hline Limitación de fumar en los restaurantes & 5,52 & 7,60 & 8,58 & 7,49 \\
\hline Limitación de fumar en los bares y cafeterías & 4,81 & 7,13 & 8,13 & 6,97 \\
\hline $\begin{array}{l}\text { Limitación de fumar en sitios públicos cerrados como } \\
\text { galerías comerciales, centros culturales, deportivos o } \\
\text { de ocio, etc }\end{array}$ & 7,64 & 8,76 & 8,85 & 8,50 \\
\hline $\begin{array}{l}\text { Establecimiento de sanciones para los responsables de } \\
\text { empresas que no hagan respetar la ley }\end{array}$ & 6,03 & 7,63 & 7,96 & 7,36 \\
\hline $\begin{array}{l}\text { Establecimiento de sanciones para las personas } \\
\text { fumadoras que no cumplan la ley }\end{array}$ & 6,26 & 7,78 & 8,01 & 7,48 \\
\hline
\end{tabular}


invocadas en el debate social y legislativo), expresadas en una escala de 1 a 10 (1 más desfavorable, 10 más favorable. No se abordan pruebas de comparación estadística dado el carácter descriptivo del estudio y los efectivos modestos de algunos subgrupos al estratificar los datos. Se presenta el grado de acuerdo y la valoración con las nueve medidas regulatorias; el grado de acuerdo se agrupa en tres categorías: desacuerdo (1-4), acuerdo (5-6), y mucho acuerdo (7-10). Se presenta también la valoración, estratificando según el patrón de uso de tabaco de los respondientes.

\section{RESULTADOS}

La población entrevistada tenía una composición por edad y sexo similar a la población catalana. Un $26,3 \%$ son fumadores diarios, mientras que los ex-fumadores son un $29 \%$. En la tabla 1 se presenta su opinión respecto al proyecto de ley. Como puede apreciarse, el grado de aceptación de la regulación era muy elevado. Los aspectos con mayor apoyo son la prohibición de la venta a menores, la prohibición de fumar en lugares públicos cerrados y centros de trabajo, y la prohibición de la publicidad. El aspecto con menor apoyo es la prohibición de fumar en bares y cafeterías. En la tabla 2 se presenta la valoración de estas medidas según el status tabáquico del entrevistado. Aunque los fumadores expresan menos apoyo que los no fumadores, las medidas regulatorias son mayoritariamente aceptadas por los fumadores: tan solo reciben un apoyo menor las limitaciones en bares y cafeterías.

\section{DISCUSIÓN}

Los resultados muestran que la regulación planteada por la Ley 28/2005 recibe un apoyo muy mayoritario de la población. Esto es importante, y conocerlo durante el proceso legislativo permite reforzar el pro- ceso y debilita los argumentos de los agentes contrarios. Este tipo de iniciativas, frecuentes en el debate político general y en los debates sanitarios en otros países, son poco habituales en nuestro medio en relación a las políticas de salud ${ }^{6}$. Quizás tenemos cierta tendencia idealista a considerar que las políticas sanitarias se relacionan de forma directa con las aportaciones de la ciencia sin interferencias del contexto político. Lo cierto es que los grupos de interés, la posición de los medios de comunicación, y la ideología median de forma considerable en su desarrollo ${ }^{7}$. Hacer emerger la expresión de la opinión pública puede ejercer una influencia decisiva a favor de la salud en momentos cruciales. Una estrategia de salud pública orientada a vencer las resistencias a la formulación de políticas favorables a la salud puede y debe tenerlo en cuenta. Los esfuerzos realizados por el movimiento de prevención para cambiar la visión del tabaco en los medios de comunicación van en este sentido ${ }^{8}$. En este caso las autoridades sanitarias desarrollaron diversas acciones orientadas a crear un clima favorable a la regulación del tabaquismo en Cataluña, entre ellas la realización y difusión de esta encuesta.

Hay precedentes de este tipo de estudio, aunque sin aplicaciones similares. En años anteriores se había documentado un nivel elevado de apoyo a la regulación por la población catalana, y nos consta que la administración central también exploró el grado de apoyo social a la regulación durante el proceso, aunque no difundió los datos $^{9,10}$. En nuestro caso se dio el uso deliberado de una información obtenida con rigor metodológico para alimentar a los medios de comunicación, como parte de un conjunto de acciones planificadas para construir un clima social orientado a la prevención.

El contexto de aprobación de la ley no se puede comprender sin conocer los cambios en la epidemiología del tabaquismo ${ }^{11}$. En 
los últimos años, la disminución de la prevalencia en los varones y la emergencia de un segmento importante de ex-fumadores jóvenes contribuyen a crear un clima propicio a la regulación, de forma congruente con el esquema planteado para analizar la epidemia tabáquica ${ }^{12}$. Pero además, la difusión de información y la educación del público hacen que un segmento importante de los fumadores sean disonantes y acepten de buen grado la regulación. Frente a quienes plantean que los fumadores se oponen a la regulación y por tanto que las políticas de control del tabaquismo generan controversia y conflictos, la encuesta muestra el amplio apoyo social de la regulación, al que contribuyen muchos fumadores. Por otra parte, hay datos que muestran como en ausencia de una regulación rigurosa la exposición al humo ambiental es general y genera amplia frustración, incluso en espacios presuntamente libres de humo ${ }^{13}$. La aplicación de la ley, mayoritariamente aceptada y libre de incidentes, es un resultado de este estado de opinión. Aunque la industria tabaquera utiliza la controversia para retrasar la regulación, ésta genera un amplio apoyo social ${ }^{14}$. Sería de gran interés documentar el impacto de la regulación en aspectos como la presión publicitaria o la exposición al humo ambiental de tabaco para evaluar los efectos positivos de la regulación, a veces poco visibles ${ }^{15,16}$. Esta experiencia puede quizás extrapolarse a otras situaciones en que los avances de la regulación en pro de la salud pública han de enfrentarse también a la resistencia de algunos grupos de interés.

\section{AGRADECIMIENTOS}

Este trabajo se ha realizado en el marco de la acción estratégica de evaluación de intervenciones y servicios de la red de centros de investigación en epidemiología y salud pública (RCESP C03/09) del FIS. La realización de la encuesta se ha financiado con fondos del Departament de Salut.

\section{BIBLIOGRAFÍA}

1. Subirats J. El análisis de las políticas públicas. Gac Sanit 2001; 15: 259-64.

2. Villalbí JR, López V. La prevención del tabaquismo como problema político. Gac Sanit 2001; 15: 265-72.

3. Asbridge M. Public place restrictions on smoking in Canada: assessing the role of the state, media, science and public health advocacy. Soc Sci Med 2004; 58: 13-24.

4. Boletín Oficial de Estado. Ley 28/2005 de 26 de diciembre, de medidas sanitarias frente al tabaquismo y reguladora de la venta, el suministro, el consumo y la publicidad de los productos del tabaco. BOE 309 de 27/12/2005; 42241-50.

5. Granero L, Villalbí JR, Gallego R. ¿Quién se opone a la prevención? Un mapa de los actores pro-tabaco en España. Gac Sanit 2004; 18: 374-9.

6. Chapman S, Lupton D. The Fight for Public Health: Principles and Practice of Media Advocacy. London: BMJ Books, 1994.

7. Villalbi JR, Córdoba R. El control del tabaquismo y el movimiento de prevención. Adicciones 2005; 17: $297-300$

8. Observatorio de la Comunicación Científica y Médica de la Universitat Pompeu Fabra. Medicina, comunicación y sociedad. Informe Quiral 1999. Barcelona: Rubes Editorial, 2000; 121.

9. Salleras L, Pardell H, Jané M, Saltó E, Cabezas C, Marín D et al. Dona i tabac. Informe tècnic sobre l'hàbit tabàquic femení a Catalunya. Barcelona: Departament de Sanitat i Seguretat Social, Generalitat de Catalunya, 2001 (disponible en: http:// www.gencat.net/salut/depsan/units/sanitat/pdf/don atabac1.pdf).

10. Jané M, Pardell E, Saltó E, Salleras L Epidemiología del tabaquismo femenino. Factores determinantes de la iniciación y del mantenimiento. Prev Tab 2001; 3: 147-154.

11. Banegas JR, Díez-Gañan L. Epidemiología del tabaquismo. Morbimortalidad. En: Jiménez-Ruiz C, Fagerstrom KO. Tratado de tabaquismo. Madrid: Aula Médica 2004; 11-27.

12. Lopez AD, Collishaw NE, Piha T. A descriptive model of the cigarette epidemic in developed countries. Tobacco Control 1994; 3: 242-247.

13. Font-Mayolas S, Planes M, Gras ME. Consumo de tabaco en el lugar de trabajo: interacciones entre 
fumadores y no fumadores respecto al cumplimiento de la normativa. Oncología 2006; 29: 93-4.

14. Saloojee Y, Dagli E. Tobacco industry tactics for resisting public policy on health. Bull World Health Organ 2000;78: 902-10.

15. Gallus S, Zuccaro P, Colombo P, Apolone G, Pacifici R, Garattini S, La Vecchia C. Effects of new smoking regulations in Italy. Ann Oncol 2006; 17 : 346-7.

16. Lopez MJ, Nebot M, Salles J, Serrahima E, Centrich F, Juarez O, Ariza C. Medición de la exposición al humo ambiental de tabaco en centros de enseñanza, centros sanitarios, medios de transporte y lugares de ocio. Gac Sanit. 2004; 18: 4517. 